\title{
Guest editorial: Special issue-revised selected papers of the LION 5 conference
}

\author{
Carlos A. Coello Coello
}

Published online: 12 November 2013

(C) Springer Science+Business Media Dordrecht 2013

The International Conference on Learning and Intelligent OptimizatioN (LION) has as its goal to explore the intersections between machine learning, artificial intelligence, mathematical programming and algorithms for hard optimization problems. The main purpose of this conference is to bring together experts from these disciplines, in order to discuss new ideas and methods, as well as to identify challenges and opportunities in various application areas, general trends and specific developments.

This special issue contains the extended and revised versions of three carefully selected papers that were presented at LION 5, which took place in Rome, Italy, during January 17-21, 2011.

The first paper, from Sato, Aguirre and Tanaka, presents a study of the effectiveness of crossover and mutation operators in 0/1 knapsack problems having four or more objectives and up to 20 items. The authors show that recombining very dissimilar individuals, without setting a limit to the information being crossed, causes the operator to become too disruptive and, consequently, decreases its effectiveness. In order to deal with this problem, the authors propose to use a local recombination operator that selects mating parents based on proximity in objective function space as well as two-point and uniform crossover operators that control the maximum number of crossed genes. These operators are shown, through a series of experiments, to significantly improve performance.

The second paper, from Kampouridis, Alsheddy and Tsang, presents a thorough study of different hyper-heuristics frameworks, with the aim of improving the performance of a financial forecasting tool, based on genetic programming, called EDDIE 8. The authors devised 14 heuristics, which were applied to 30 different

C. A. Coello Coello $(\bowtie)$

Department of Computer Science (Evolutionary Computation Group),

CINVESTAV-IPN, Av. IPN No. 2508, Col. San Pedro Zacatenco, México, 07360, México

e-mail: ccoello@cs.cinvestav.mx 
datasets. Then, they selected 10 heuristics, which were tested in three different hyperheuristics frameworks. The three hyper-heuristics adopted by the authors were able to obtain competitive results, significantly outperforming the original algorithm.

The third paper, by Martí, García, Berlanga and Molina, presents as its main hypothesis that error-based learning, which is the most commonly used learning adopted in multi-objective optimization estimation of distribution algorithms (MOEDAs) is responsible for their poor performance. The authors propose the use of adaptive resonance theory as an alternative learning paradigm alternative. The approach proposed by the authors, adopts a Gaussian adaptive resonance theory neural network for model-building and a hypervolume-based selection mechanism. This approach is shown to outperform other MOEDAs as well as several modern multi-objective evolutionary algorithms.

Carlos A. Coello Coello

Technical Program Committee Chair, LION 5 